\title{
LA PERSONALIZACIÓN DE LAS ZAPATILLAS DE BALONCESTO
}

\author{
Martínez, Jose A. ${ }^{1}$ \\ Departamento de Economía de la Empresa \\ Universidad Politécnica de Cartagena \\ josean.martinez@upct.es
}

Material original autorizado para su primera publicación en la revista académica REDMARKA. Revista Digital de Marketing Aplicado.

https://doi.org/10.17979/redma.2013.01.010.4757

Recibido: 16 Mayo 2013

Aceptado 15 Junio 2013

\section{RESUMEN}

Esta investigación describe las diferentes formas en las que un jugador de baloncesto puede personalizar sus zapatillas, mostrando asimismo los diversos tipos de mensajes que en éstas se pueden transmitir, y relatando ejemplos concretos de cada una de esas categorías. Así, este manuscrito recoge las diversas tipologías de personalización que existen en el baloncesto actual, distinguiendo entre las controladas por las marcas y las que son sólo responsabilidad del jugador. Además se recapitula el gran número de opciones que existen de personalización, ofreciendo a las marcas una coyuntura para encontrar oportunidades de negocio tras el análisis de cómo ciertos jugadores escriben mensajes en sus zapatillas.

${ }^{1}$ Profesor Contratado Doctor del Departamento de Economía de la Empresa de la Universidad Politécnica de Cartagena. Entre sus intereses de investigación destacan el comportamiento del consumidor, el marketing de servicios y la metodología de investigación. Ha publicado más de medio centenar de artículos en revistas académicas, entre ellas: Tourism Management, Journal of Operations Management, Journal of Sport Management, Methodology, Management Decision, Managing Service Quality, Journal of Business Research o Journal of Retailing and Consumer Services. 
PALABRAS CLAVES: marketing, personalización, zapatillas de baloncesto

\section{ABSTRACT}

THE CUSTOMIZATION OF BASKETBALL SHOES

This research shows the different forms basketball players may customize their shoes and the divergent types of messages which can be transmitted from them. In addition, several examples of all these categories are explained. Therefore, this paper depicts typologies of customization, distinguishing customization planned by shoe brands from customization planned only by players. Moreover, explanations of each category of customization may help brands to find opportunities for business through the analysis of how some players write messages in their shoes.

KEYWORDS: marketing, customization, basketball shoes, 


\section{INTRODUCCIÓN}

Las zapatillas de baloncesto constituyen actualmente uno de los ejemplos más interesantes de producto deportivo personalizado, tanto dentro como fuera del deporte profesional. Las marcas son conscientes de la importancia de la línea de productos para este deporte, debido a los altos márgenes de beneficio por unidad, y a que son todo un exponente de la cultura urbana (Brace-Govan y Burgh-Woodman, 2008), especialmente de la subcultura hip-hop, (Styles, 2005) y como modo de expresión de clases sociales más desfavorecidas (Vanderbilt, 1998). Según Rovell (2008), cuatro de cada cinco personas que compran zapatillas de baloncesto no practican este deporte habitualmente; la industria del baloncesto-moda es un negocio de 2 billones de dólares. Por tanto, las zapatillas de baloncesto son un producto de alto nivel estratégico para los fabricantes y distribuidores de calzado deportivo.

Este producto ha trascendido su utilización más allá del mero beneficio técnico que supone para el deportista, siendo un vehículo de expresión de la identidad del individuo que lo consume, incluso si ese consumo es realizado únicamente para el baloncesto profesional. De este modo, el valor simbólico (Belk, 1988) de este producto hace que las marcas diseñen diferentes estrategias de marketing basadas en un doble uso del concepto de personalización: (1) la personalización de los modelos para los jugadores de baloncesto profesionales; (2) la personalización para el gran público. En el primer caso, las marcas visten de manera individualizada a las grandes estrellas de este deporte, con modelos únicos que enfatizan características distintivas de la identidad del jugador y que pueden llegar a transmitir aspectos muy personales de éste. En el segundo caso, las nuevas tecnologías permiten en la actualidad la personalización de diferentes modelos de marcas como Nike, Converse, Adidas o Reebok, tanto a nivel de colores, estampados o grabación de mensajes. Así, por ejemplo, cuando un consumidor compra un modelo firmado por un jugador profesional, está transmitiendo los valores, la imagen y las características que proyecta ese jugador, por lo que existe una transferencia de significado (Dimofte y Yalch, 2011; Stallen, Smidts, Rijpkema, Smit, Klucharev y 
Fernández, 2010), esto es, una forma de identificación con él y de comunicación social a la vez.

Pero hay formas divergentes de personalización que los jugadores profesionales utilizan. Por ejemplo, ciertos jugadores colaboran con las marcas para diseñar zapatillas que lleven distintivos personales. En otras ocasiones, algunos jugadores realizan personalizaciones al margen de las marcas, y es cuando utilizan sus zapatillas como vehículo de expresión de mensajes que en momentos puntuales quieren transmitir. En esos casos, el jugador, de manera espontánea, personaliza sus zapatillas utilizando diversos instrumentos para pintarlas y así lanzar el mensaje oportuno. Esa acción normalmente refleja un momento psicológico de efervescencia, donde el jugador necesita expresar un mensaje que se haga eco de un estado motivacional elevado, provocado por las más divergentes circunstancias, como a continuación se expondrá.

La importancia del concepto de personalización es indiscutible en el marketing actual (Vesanen, 2007), porque los consumidores demandan productos únicos que los hagan diferenciarse del resto, y al mismo tiempo que reafirmen y proyecten su identidad social e individual, es decir, su definición a partir de sus rasgos únicos e idiosincrásicos (Morales, Moya, Gaviria y Cuadrado, 2007). Las marcas conocen perfectamente que la relación estrecha y única con los jugadores es un factor clave en la fidelidad de éstos, a veces, independientemente del factor económico. Por ejemplo, Michael Jordan firmó por Nike en 1984 en lugar de por Adidas (su marca preferida), porque, además de la clara diferencia económica en el contrato ofrecido, Nike le brindaba la oportunidad de establecer una relación estrecha y única, con un producto completamente personalizado y en el que Jordan podía intervenir en su diseño. Ello distaba de un tratamiento más estándar que podían ofrecerle Adidas o Converse, las marcas líderes de baloncesto en aquella época (Falk, 2009). Jordan fue con las condiciones de ese contrato a hablar con Adidas, con el objetivo de que si la marca alemana se aproximaba a establecer unas condiciones similares, entonces firmaría con ellos. Pero Adidas no mejoró su oferta, y esto cambió la historia del marketing de las zapatillas de baloncesto. 
Pero, ¿cuáles son las diferentes maneras de personalizar una zapatilla de baloncesto para los jugadores de elite y qué tipos de mensajes se transmiten? Esa es la principal pregunta a la que trata de dar respuesta este estudio. Para ello, se describen las divergentes formas que las marcas y los jugadores profesionales tienen de personalizar este producto. El objetivo de esta investigación es, por tanto, profundizar en las maneras en las que se produce esa personalización de producto en el baloncesto profesional y universitario, con el fin de establecer diferentes tipologías, y de este modo, realizar un dibujo acerca de cómo las marcas utilizan a los jugadores de baloncesto para desarrollar sus estrategias de producto, y cómo también los jugadores de baloncesto utilizan ese producto para transmitir mensajes relacionados con su identidad. A través de la utilización del método mixto (O'Cathain, Murphy y Nicholl, 2008), esta investigación ha realizado un análisis bibliográfico de más de 3000 ejemplares de revistas de baloncesto publicadas en España, ha explorado webs especializadas, $\mathrm{y}$ ha entrevistado a varios jugadores profesionales, siendo pionera en esta disciplina de conocimiento en realizar un análisis sobre la relación de los jugadores con las marcas en referencia a la personalización de zapatillas. Es, de este modo, la primera investigación que trata esta temática, y que relata algunos de los más curiosos ejemplos ocurridos en el baloncesto de elite, siendo su análisis de especial interés también para la personalización de zapatillas para el gran público. Así, los resultados de este estudio muestran las diferentes formas en las que las marcas utilizan a los jugadores para el desarrollo y lanzamiento de sus productos, y ofrece una coyuntura para encontrar oportunidades de negocio, tras el análisis de cómo ciertos jugadores escriben mensajes en sus zapatillas.

\section{MARCO TEORICO}

\subsection{El calzado deportivo y las zapatillas de baloncesto}

La historia del calzado deportivo cambió cuando se empezaron a vender las primeras suelas de goma en 1876, por la New Liverpool Rubber Company, 
basado en la tecnología de vulcanización de Goodyear ${ }^{2}$. Poseriormente, en 1892, la U.S. Rubber Company, también comenzó a comercializar zapatillas con ese tipo de suela, compañía que puso a disposición del público la marca Keds en $1916^{3}$, tras unificar varias marcas de calzado en una sola. Otras compañías importantes en aquella época fueron las estadounidenses Spalding (1876) y Converse (1908), la checoslovaca Bata (1894), la inglesa Gola (1906), la japonesa Mizuno (1906), la finlandesa Karhu (1916) o la alemana Hummel (1923). Mientras, la empresa que posteriormente se conocería por Reebok, emergió en 1890 en Inglaterra, gracias a Joseph William Foster, quien diseñó los primeros zapatos de running con clavos (www.reebok.com). El mercado de las zapatillas deportivas comenzó su segundo gran cambio en los años 20, cuando los hermanos Adolf y Rudolf Dassler abrieron su primera tienda de zapatos para jugar al fútbol en Herzogenaurach, Alemania. Después de la Segunda Guerra Mundial, y tras su archiconocida pelea (ver Smit, 2007), Aldof fundó Adidas (1949) y Rudolf creó Puma (1948), marcas que, junto a Nike ${ }^{4}$ (1972), revolucionaron la industria.

Las zapatillas deportivas también se conocen como "sneakers", término que se comenzó a acuñar cuando la gente se dio cuenta de que las suelas de goma no hacían ruido al pisar ${ }^{5}$, siendo las principales responsables de la acuñación de este término las zapatillas de lona Keds y Converse All-Star, que pronto se hicieron muy famosas en Estados Unidos. Aunque esas zapatillas se usaban para realizar deporte, actualmente las zapatillas para atletas contienen poca o ninguna goma, como indica Vanderbilt (1998), y se usa espuma de poliuretano y EVA para las suelas, empleando nylon, cuero o materiales sintéticos para la parte de arriba de la zapatilla. Así, la industria ha adoptado diferentes

\footnotetext{
${ }^{2}$ La vulcanización es un proceso por el que se calienta el caucho (goma) en presencia de azufre, para convertirlo en un material más duro y resistente. Fue descubierto por Charles Goodyear en 1839 al volcar accidentalmente un recipiente de azufre y caucho sobre una estufa.

${ }^{3}$ Otras fuentes señalan el año 1917

${ }^{4}$ Aunque la marca Nike se creó en 1972, la empresa ya existía como Blue Ribbon Sports desde los años 60, distribuyendo en Estados Unidos zapatillas de running japonesas creadas por Onitsuka, y que gracias a la habilidad en los negocios de Phil Knight, y del trabajo de mejora de esas zapatillas realizado por el entrenador de atletismo Bill Bowerman, consiguieron muy pronto una gran aceptación por parte de los atletas americanos. La historia de Nike puede consultarse en Frish (2009), quien sitúa la creación de Blue Ribbon Sports en 1962, tras el primer viaje a Japón de Phil Knight.

5 "Sneaky" significa sigiloso en inglés.
} 
categorías para cada tipo de zapatilla. En cualquier caso, las zapatillas de baloncesto suelen tener goma en la suela exterior (outsole), es decir, la que hace contacto con el suelo.

No resulta fácil dar cifras fiables sobre el negocio de las zapatillas, y es que es difícil delimitar el propio concepto de zapatilla de deporte. Las zapatillas con suela de goma y lona, como muchos modelos de Converse, se venden en tiendas de zapatillas para atletas, como Foot Locker o Finish Line, pero obviamente no están pensadas para hacer deporte. No obstante, algunas fuentes señalan cifras muy elevadas de negocio, como los 350 millones de pares vendidos al año en Estados Unidos (DeMello, 2009), dato que concuerda con el que indica Vanderbilt (1998) referido al año 1997. La National Shoe Retailers Association y U.S. Census Bureau (http://www.statisticbrain.com/footwear-industry-statistics/) muestran también cifras muy importantes de ventas en distribuidores especializados como Foot Locker, donde en el año 2010 facturó sólo en Estados Unidos 4.8 billones de dólares en sus 4000 tiendas.

Según la consultora estadounidense Packaged Facts, en su estudio de 2009 (Packaged Facts, 2009), por zapatillas para atletas se entiende zapatillas para atletas profesionales, deportistas aficionados, y personas que las usan para practicar un estilo de vida saludable. Incluye además aquellas zapatillas de suela de goma inspiradas en las zapatillas para hacer deporte pero que se usan para otras actividades (caminar, vestir casual, etc). También se consideran botas de escalada, sandalias o botas de esquí. Sobre esta definición, la consultora provee diversas cifras de interés.

Por ejemplo, Nike, Adidas, Puma y Asics ocupan los 4 primeros puestos en ventas de calzado deportivo a nivel mundial en 2008, con 10894, 7237, 2110 y 1728 millones de dólares, respectivamente. Hay que destacar que estas marcas representan conglomerados de empresas de la misma propiedad. Por ejemplo, Nike incluye a Jordan, Cole Haan, Converse, Hurley, Nike Golf, y Umbro. Mientras que Adidas incluye a Reebok, empresa que adquirió en 2006. 
Las zapatillas deportivas constituyen el principal negocio de Nike: un 54\% de la facturación global de la marca en el año fiscal de 2009 (de mayo 2008 a mayo 2009), es decir, la línea de calzado pesa más que la de textil. Under Armour consigue unas cifras similares $(52 \%)$, mientras que para Adidas el calzado deportivo representa el $46 \%$ de sus ingresos.

De acuerdo con Packaged Facts, el 45\% del calzado que se vendió en Estados Unidos en 2008 eran zapatillas deportivas, es decir, hay prácticamente equilibrio entre el calzado casual, de vestir y de trabajo, y el deportivo. Sin embargo, si se miran sólo al segmento masculino, las ventas de calzado deportivo casi duplican al resto (10194 millones de dólares frente a 6807). Esto indica que para los hombres, las zapatillas de deporte constituyen su forma principal fuente de gasto en la categoría de calzado. En cuanto a segmentos de edad, también existe disparidad. Así, en 2001, y según Yoh (2005), 25 millones de adolescentes entre 11 y 17 años gastaron 3.4 millones de dólares en calzado deportivo en Estados Unidos, casi un 25\% de las ventas totales. Y es que el adolescente americano medio compra unos 10 pares de zapatillas deportivas al año (Slater y Lloyd, 2002), tendencia que se mantiene desde los años 70 (Strasser y Becklund, 1991). Entre los jugadores profesionales la pasión por las zapatillas tiene un carácter heterogéneo, habiendo jugadores que las ven como meramente un instrumento que le ayuda en su trabajo (ej. Andrés Jiménez), o que las consideran como un objeto de culto, formando colecciones de cientos o miles de zapatillas (ej. Joe Johnson, Nacho Martín).

Dentro de la línea de calzado deportivo, la zapatilla de baloncesto ocupa un lugar muy importante. Para entender la relevancia de este producto hay que comenzar con algunas cifras globales. Por ejemplo, según la Federación Internacional de Baloncesto (FIBA), más de 450 millones de personas practica este deporte, entre la competición profesional y el baloncesto de base, datos $\begin{array}{llll}\text { referidos } & \text { al } & \text { año } & 2007\end{array}$ (http://www.fiba.com/pages/eng/fc/FIBA/quicFact/p/openNodelDs/962/selNodel D/962/quicFacts.html). Además, más de un 10\% de la población mundial juega al baloncesto. En países como Francia, Reino Unido, España y Alemania, existen conjuntamente unos 33 millones de aficionados al baloncesto, con un 
perfil principalmente joven (14-24 años), de sexo masculino y de un estatus social acomodado. En Estados Unidos, según la Sporting Goods Manufacturers Association (Sfia.org, 2008), en 2008 había cerca de 26 millones de practicantes, siendo el número de aficionados varios millones mayor. El perfil tipo del practicante coincide con el estudio realizado en Europa: jóvenes de clase media-alta y que viven en grandes áreas metropolitanas.

Por tanto, existe un alto y creciente interés por el baloncesto en prácticamente todo el mundo. Eso hace que el consumo de productos necesarios para la práctica de este deporte (canastas y accesorios) genere un negocio de 353 millones de dólares. Sin embargo, las ventas globales zapatillas de baloncesto superan con creces la anterior cifra, siendo en 2007 superior a 1 billón de dólares. Y es que sólo el modelo Air Force One de Nike vendió 10 millones de pares en Estados Unidos en 2011 (Seize9, 2012d).

Hay que resaltar, asimismo, que otras categorías de calzado deportivo, como las zapatillas de walking, running o cross training obtienen ventas superiores a las de baloncesto, según los últimos datos de 2011. No obstante, aunque las zapatillas de baloncesto no son la categoría líder en ventas, ocupan una posición privilegiada dentro del universo del calzado deportivo, industria que, como acabamos de ver, factura miles de millones de dólares anualmente.

\subsection{Las zapatillas de baloncesto personalizadas por la marca}

Históricamente, la primera forma de personalización en zapatillas de baloncesto se refiere a los modelos realizados específicamente para jugadores de elite, incluyendo algún tipo de simbología que apela a la identidad del jugador (nombre, apodo, logotipo, etc.). Desde las marcas pioneras en realizar este tipo de modelos, como Converse con el modelo Chuck Taylor All-Star en 1923, y Kinneys con su modelo Joe Lapchick en la década de los 30, numerosas empresas han lanzado al mercado los modelos con los que vestían de manera personal a sus jugadores. Tras los pioneros Taylor y Lapchick, los mejores jugadores de la NBA fueron apadrinando marcas durante las siguientes décadas (Bob Cousy, Pete Maravich, Jerry West, Rick Barry, 
Kareem-Abdul Jabbar, Julius Erving, Magic Johnson, Larry Bird...) hasta llegar a 1984 y la firma de Michael Jordan con Nike, que cambió la historia de la industria de calzado y ropa deportiva en el mundo (Falk, 2009). No obstante, los primeros modelos personalizados para jugadores NBA (también llamados pro-model) fueron realizados por Adidas para Kareem Abdul Jabbar en 1976, con un retrato en la lengüeta y su nombre grabado en la zapatilla (Smit, 2007), y por Puma para Walt "Clyde"6 Frazier en 1974 (Vanderbilt, 1998). Además, David Thompson, tras su llegada a la ABA en 1975 y a la NBA en 1976 firmó un contrato con una pequeña empresa de Baltimore: Superpro, la cual diseñó zapatillas con sus iniciales "DT".

En el baloncesto español, por su parte, en $1975^{7}$ los jugadores Emiliano Rodríguez y Nino Buscató comenzaron a realizar anuncios publicitarios de la marca Wamba, comercializada por la empresa riojana Fernández Hermanos. Esta empresa había adquirido la marca a la italiana Pirelli, la cual había comercializado la marca desde que se estableció en España en 1934. Sin embargo, no fue hasta la década de los 80 cuando los jugadores comenzaron a tener su propio modelo. Así por ejemplo, Juan Antonio San Epifanio (Epi), hasta el año 1984 utilizaba las zapatillas que le regalaban las marcas o el club (Converse, Adidas, Karhu o Superga de Pirelli eran las más comunes). A partir de esa fecha, y con el boom del baloncesto que emergió en aquellos años, Adidas contactó con Epi para que utilizara sus zapatillas ligándolo a un contrato, el cual le obligaba a utilizar las zapatillas en todos los entrenamientos y partidos durante la temporada, a cambio de material deportivo cuantificado y una contraprestación económica (contrato fijo más royalties). Epi y Adidas lanzaron su propio modelo ${ }^{8}$, al igual que ocurrió con Kelme y Jordi Villacampa en 1985 (el jugador antes calzaba modelos no personalizados de Adidas).

\footnotetext{
${ }^{6}$ Como bien explica Seize9 (2012e), Walt Frazier fue conocido por lo que hacía dentro de las canchas tanto como por lo que hacía fuera. Su estilo jugando tenía mucho que ver con la fantasía, pero su verdadero estilo se mostraba fuera. Le llamaban "Clyde" porque solía llevar un sombrero como el que llevaba Warren Beatty en "Bonnie and Clyde".

${ }^{7}$ A comienzos de los años 50 marcas como Chirucas y posteriormente Eya y Amigo, calzaban a la mayoría de jugadores en España, época aún en la que muchas pistas eran de tierra. Luego llegarían las PF Flyers, John Smith y las Converse Chuck Taylor All-Star. No obstante, no hay constancia de ningún apadrinamiento por parte de jugadores de baloncesto.

${ }^{8}$ Esas zapatillas se producían en Illueca (Zaragoza), ya que era muy caro importarlas en aquella época.
} 
Ambos firmaron contratos que fueron renovándose hasta el final de su carrera deportiva. Otros ejemplos coetáneos en el baloncesto europeo fueron la firma del jugador italiano Dino Meneghin con Sergio Tacchini y la del yugoslavo Drazen Petrovic y el italiano Walter Magnifico con Kronos, que también lucieron sus propios modelos de zapatillas.

En la actualidad, la nómina de los jugadores de baloncesto con sus propios modelos personalizados de la marca que apadrinan es enorme, e incluye, entre otros, a jugadores norteamericanos como Kobe Bryant, LeBron James y Kevin Durant (Nike), Chris Paul y Carmelo Anthony (Jordan), Derrick Rose y Dwight Howard (Adidas), franceses como Joaquim Noah (Le Coq Sportif) o Tony Parker (Peak), argentinos como Luis Scola (Anta), rusos como Andrei Kirilenko (Nike), italianos como Danilo Gallinari (Reebok), inlgeses como Luol Deng (Nike), españoles como Jose Calderón (Li-Ning) o Pau Gasol (Nike).

\subsection{La expresión de la identidad del jugador de baloncesto}

Algunos jugadores necesitan expresar los valores que reflejan su identidad a través de diferentes simbologías más allá de los posibles acuerdos explícitos sobre personalización de zapatillas que convengan con sus marcas de material deportivo.

Una de esas formas de expresión es a través de la elección del número con el que está impresa su camiseta. Por ejemplo, Kevin Durant lleva el número 35 en memoria de su entrenador Charles "Big Chucky" Craig, quien le ayudó en su formación en sus primeros años como jugador (lo conoció con 8 años), y que fue asesinado en 2005, a la edad de 35 años, por intentar para una pelea callejera entre dos jóvenes (García, 2012). Gilbert Arenas, por su parte, quiso llevar el número 0 para demostrar a aquellos que predecían que iba a jugar cero minutos en su universidad se equivocaban, mientras que Grant Hill eligió el número 33 por ser el número que Magic Johnson llevó en la universidad. Otro ejemplo es Marcus Banks, quien eligió el número 3 por su significado religioso (padre, hijo y espíritu santo), y su asociación a los rezos que hace antes de cada partido (Summers, 2013). 
Pero la forma de expresión más importante es proyectada a través de los tatuajes. Por medio de la grabación en su piel de símbolos, frases y dibujos diversos, los jugadores expresan cómo se definen a sí mismos, cuáles son sus motivaciones y recuerdos, y al mismo tiempo lanzan un mensaje sobre cómo desean ser percibidos. Como indican Fimin, Tse, Foster y Angelini (2008), los tatuajes ayudan a las personas que los llevan a auto-aceptarse y a percibir que los demás los aceptan. En la NBA, más de la mitad de sus jugadores lleva tatuajes (http://nbatattoos.tumblr.com/), porcentaje mayor que el de personas de una edad similar que llevan al menos un tatuaje en Estados Unidos (entre el 36

y el $\quad 40 \%$ según

http://pewresearch.org/databank/dailynumber/?NumberlD=927). En la Liga ACB en España, no existen datos al respecto, pero algunos jugadores cifran en torno a un $60-70 \%$ los jugadores americanos que van tatuados, mientras que los españoles son únicamente un 10\% (N. Martín, comunicación personal, Octubre 17, 2011).

Nacho Martín, jugador del CB Valladolid de la Liga ACB, ilustra perfectamente el sentido de los tatuajes para el jugador. Martín tiene más de una docena de tatuajes en su cuerpo:

“...me recuerdan cosas que he pasado y que al mismo tiempo me gusta recordar, por ejemplo, tengo el apodo de mi padre tatuado en la muñeca derecha "Morty", y cada vez que lo veo su imagen salta en mi cabeza, ese es el motivo, recordar a mi padre cada día, en cada instante que vea su nombre escrito en mi piel. También llevo las iniciales de mi madre y hermana en la mano izquierda, otra manera de tenerlas siempre presentes. En mi antebrazo izquierdo llevo tatuado el nombre de mi grupo "B-Squad" y una fecha importante para nosotros en la muñeca, me recuerda que son mis hermanos y aunque estemos separados siempre los llevo conmigo. $Y$ así un largo etcétera, son muchos tatuajes y muchas razones. ¿Si me hacen jugar mejor? Pues no, no lo creo, simplemente me traen buenos recuerdos a la mente y mantienen vivo el sentido de por qué me los hice. Está claro que cada uno busca la 
motivación como más le conviene, a mi me encanta tener a mi gente tatuada en mi cuerpo, así donde esté yo, están ellos......." (N. Martín, comunicación personal, Octubre 17, 2011).

Una gran muestra del significado de los tatuajes para los jugadores de la NBA puede encontrarse en http://nbatattoos.tumblr.com/. Como se desprende de esa fuente, la diversidad de mensajes que representan es enorme (versículos de la biblia, homenaje a familiares o amigos, palabras o frases motivadores, símbolos del baloncesto, etc.), aunque a veces un tatuaje pueda ocasionar a un jugador un contencioso con una marca deportiva. Tal fue el caso del jugador polaco Marcin Gortat, quien rompió su vinculación con Reebok en 2009 porque esta marca le pidió que borrara (o al menos que camuflara) el tatuaje que el jugador tiene en la pantorrilla: "The jumpman", es decir, la silueta de Michael Jordan. Ante la negativa de éste, ambas partes finalizaron su relación y el jugador firmó subsecuentemente con Nike. Esta anécdota ilustra el gran valor que algunos jugadores otorgan a sus grabados en la piel.

Sin embargo, esos tatuajes tienen carácter permanente (aunque eventualmente pueden borrarse), es decir, el jugador los lleva siempre en todos los partidos. Pero hay ocasiones en los que el jugador necesita transmitir un mensaje puntual, algo relacionado con un evento específico. En fútbol, en las últimas dos décadas los jugadores utilizan camisetas interiores donde llevan inscrito el mensaje que desean transmitir, y que normalmente hacen público si consiguen un gol. No obstante, las reglas actuales de la máxima competición en España prohíben ya esas acciones. Así, el artículo 91 del reglamento relativo a las celebraciones dice textualmente que quien "alce la camiseta y exhiba cualquier clase de publicidad, lema, leyenda, siglas, anagramas o dibujos, sean los que fueren sus contenidos o finalidad, será sancionado con una multa en cuantía de 2.000 a 3.000 euros y amonestación". Y en baloncesto, la situación es similar y, dadas las reglas sobre uniformes, el único resquicio que le queda al deportista es utilizar sus zapatillas para tal fin. A este respecto, las normas no suelen imponer límites, aunque es cierto que en determinados momentos se han llegado a establecer ciertas reglas sobre el uso de las zapatillas, como la 
limitación del uso del color rojo en los 80 en la NBA (algo que utilizó Nike con su primer modelo Air Jordan para conseguir una publicidad impagable pese a la multa de 1000 dólares que pagaba el jugador por llevar esas zapatillas en cada partido), o la normativa que en 2004 aprobó la Liga ACB por la cual el color de las zapatillas de los jugadores del mismo equipo debía ser uniforme. La multa por no hacerlo era de 150 euros. Ambas normas fueron abolidas poco tiempo después.

Por tanto, el jugador puede utilizar sus zapatillas como medio de comunicación, como hace con sus grabados en la piel. Es, en cierta manera, tatuar sus zapatillas. En este sentido, la tecnología actual permite diferentes tipos de personalización, que proporciona al jugador (ya sea profesional o aficionado) un abanico amplio de posibilidades de llevar un producto único que ayude a expresar sus valores.

\section{METODOLOGÍA}

A la hora de establecer las tipologías de personalización se utilizaron tres fuentes de información diferentes: publicaciones periódicas especializadas, internet y entrevistas a jugadores, empleando el método mixto de investigación (O'Cathain, Murphy y Nicholl, 2008).

En cuanto a las publicaciones periódicas, se visionaron una a una las alrededor de 176500 páginas (unas 70 de media por número) de 3122 ejemplares de revistas y publicaciones de baloncesto de tirada nacional en España desde el 1 de enero de 1980 hasta el 31 de diciembre de 2011. La población de publicaciones es de 3198 , por lo que la muestra comprende más del $97,6 \%$ del total.

En la población de estudio se incluyen soportes específicos de baloncesto con formato revista, periódico o coleccionable. Además, se incluyen las publicaciones digitales, es decir, archivos en formato revista que se pueden descargar de internet. Para ello, se utilizaron colecciones particulares de esas publicaciones periódicas, búsqueda en internet, y el archivo de la Fundación 
Pedro Ferrándiz, que contenía la mayor biblioteca del mundo de este deporte. En el Apéndice 1 se describen las características de la muestra de publicaciones periódicas utilizada.

En cuanto a internet, se realizó una búsqueda general en buscadores sobre la personalización de zapatillas, y se inspeccionaron webs y blogs especializados como: $\quad$ www.suelasdegoma.com, http://seizenine.blogspot.com/, www.nicekicks.com, www.sneakerfreaker.com, www.lovezapas.es.

Finalmente, se realizaron varias entrevistas a jugadores profesionales en activo o retirados con el fin de se pudiera cubrir un amplio espectro temporal: Nino Buscató, Roger Esteller, Aíto García Reneses, Óscar González, Andrés Jiménez, Jose Luis Llorente, Juanma L. Iturriaga, Nacho Martín, Ferrán Martínez, Juan A. San Epifanio (Epi) y Luis Miguel Santillana. Las entrevistas se efectuaron durante 2010, 2011 y 2012, y en ellas los entrevistados comentaron diversas experiencias sobre las zapatillas de baloncesto y su relación con las marcas que los vestían.

Una vez recopilada toda la información de revistas, internet y entrevistas a jugadores, se siguieron los principios metodológicos del análisis de contenido (Riffe, Lacy y Fico, 2008) para codificar las apariciones de formas de personalización. De acuerdo a Neuendorf (2002) se propusieron varias categorías a priori pero con la flexibilidad de poder añadir categorías si estás emergían del análisis, combinando la aproximación deductiva con la flexibilidad del método inductivo, concordancia con el estudio de Kelly y Tian (2004).

\section{RESULTADOS}

\subsection{Formas de personalización controladas por la marca}

Existen diversas formas en las que las marcas personalizan su producto para los jugadores profesionales (y por tanto, controlan directamente esa personalización) que pueden resumirse en las siete categorías que se muestran a continuación: 
1. La primera categoría se refiere a la forma más primordial de personalización, donde la zapatilla tiene elementos distintivos básicos que la ligan al jugador. Sin embargo, no indican nada complejo acerca de sus cualidades o personalidad, más allá de un nombre o símbolo identificativo, que bien es cierto, puede reflejar ciertos atributos del deportista. Estos son los casos, por ejemplo, de las Adidas de Kareem Abdul-Jabbar, donde se mostraba una caricatura del jugador en la lengüeta, de las Puma de Walt Frazier, donde se mostraba su apelativo "The Clyde", o de las Nike de Michael Jordan cuando éstas incorporaron su clásica silueta "The Jumpman" en 1988, que reflejaba una acción clásica de juego del jugador. Este último símbolo, posteriormente se convirtió en el icono de la marca "Jordan" en 1997, empresa subordinada de Nike. Ejemplos más recientes son las Jordan Alpha para Jason Thompson y las Nike de André Kirilenko. Ambas llevan grabados sus apelativos: JTDAKID y AK47, respectivamente (en referencia a sus iniciales y "el Niño" -DAKID- para Thompson, y el rifle AK47 para Kirilenko).

2. La segunda categoría se refiere a cuando las zapatillas del jugador reflejan aspectos de su personalidad que van más allá de un apodo o logotipo; son como mensajes más explícitos sobre lo que el jugador quiere transmitir. Por ejemplo, las Jordan CP3 de Chris Paul, en su modelo de 2008, tenían grabado el número 61, en referencia a los puntos que consiguió Paul en un partido de instituto en homenaje a la edad de su abuelo asesinado. Además llevaban grabado las iniciales de su hermano, el nombre de sus padres y entrenador de la universidad y se fecha de cumpleaños. Otro ejemplo es el de Jose Calderón; el jugador español llevó en el modelo Yu Shuai V de la marca Li-Ning, además de su propio logo con su clásico gesto al encestar un triple, la grabación de dos palabras (una en cada zapatilla): sacrificio y humildad, que son un reflejo de los valores que el jugador transmite, de su personalidad, y por supuesto, valores que a Li-Ning le interesa también transmitir e identificarse con ellos. La quinta esencia de este tipo de personalización se produjo con el lanzamiento en 2005 de las Nike Air 
Jordan XX, con motivo de su vigésimo aniversario. Aunque Jordan estaba ya retirado ese año, se pusieron a la venta unas zapatillas donde aparecían múltiples referencias a su trayectoria personal y profesional, como por ejemplo (Seize9, 2012a): un Chevrolet Monte Carlo de 1976, con la silueta de Michael sentado detrás; Una caja de herramientas en homenaje a su padre; La leyenda "44DT" por el ídolo de Jordan: David Thompson; Un logotipo formado por las cifras 97 y 38 a los lados de una cara, recordando el llamado "Flu Game", el partido de 1997 en el que Jordan llegó a los 38 puntos con fiebre; Una caja con "AJ 3", por aquella zapatilla mítica; Las iniciales "TH" por Tinker Hatfield (el diseñador de las Air Jordan); Una mano con seis dedos, por los seis anillos; Un anillo rodeando un "6"; Spike Lee en el papel de Mars Blackmon; Un silbato formado por las letras "CH", por "Coach Herring", el entrenador que rechazó a Michael en el instituto; Una pantera negra. Su apodo en el instituto que inspiró, aún sin saberlo, las Jordan XIII; Además, siluetas de cada una de las Air Jordan anteriores, partes de ellas (como la concha de la XIV) o cifras relacionadas con Michael como el 45, dorsal de su vuelta, "55" por los puntos que anotó en New York o sus 5 MVP.

3. La tercera categoría engloba las acciones puntuales que las marcas realizan para aprovechar un momento preciso de la temporada relacionado con algún evento específico. En estas ocasiones, las marcas pueden vestir a ciertos jugadores con modelos especialmente diseñados para tal ocasión. Así, por ejemplo, eventos como el All-Star, la Navidad, Hallowen o las actividades de apoyo al cáncer de mama, son aprovechadas por las marcas para vestir a algunos jugadores con modelos diseñados sólo para esos acontecimientos. De este modo, Adidas ha vestido a Derrick Rose, Kevin Garnett y Tim Duncan con modelos para el All-Star, Navidad y Hallowen (adizero Rose 2.5, Supernatural Commander y Cut Creator, respectivamente) y Nike a LeBron James con zapatillas rosas especiales en el día en que su equipo realizó acciones de apoyo al cáncer de mama (Max LeBron VII). Estos modelos son personalizados para esos jugadores, independientemente de que esas marcas lancen también modelos 
conmemorativos de esos eventos que pueden ser llevados por otros jugadores y por el gran público, como el lanzamiento de la versión en rosa Nike Zoom Soldier III en 2009 para apoyar al cáncer de mama, 0 las Air Force One de Nike para conmemorar San Valentín en 2011.El mes de la historia negra, el día de San Patricio, el día de Martin Luther King o el día de las Fuerzas Armadas son otros eventos que las marcas emplean para lanzar modelos especiales. En otras ocasiones, las marcas implican a ciertos jugadores en acciones de apoyo a campañas solidarias, y utilizan las zapatillas como medio de transmisión de esos mensajes. Así, Kobe Bryant apoyó la campaña conjunta de Nike y (RED) contra el SIDA llevando sus zapatillas con cordones de color rojo en algún partido en 2010, algo que también hicieron varios jugadores de la liga ACB vestidos por Nike, aprovechando el enfrentamiento MadridBarcelona

4. Una cuarta categoría engloba las acciones en las que el jugador y la marca colaboran para lanzar un modelo único en un día especial, y donde se graban mensajes específicos que el jugador quiere transmitir. En este sentido, no hay una total espontaneidad por parte del jugador, sino que se planifica el diseño de esas zapatillas para que lleguen grabadas al partido indicado. Casos destacados son los de Tracy McGrady y Kevin Garnett, quien el día de las elecciones en Estados Unidos en 2008, aprovechando que jugaba Houston contra Boston, ambos llevaron modelos de Adidas con las frases grabadas "Change is needed. Vote '08" y "Embrace Change. Vote '08", respectivamente. Ambas frases fueron elegidos por esos jugadores para reflejar sus ideas políticas, y estaban en consonancia con los mensajes emitidos por Obama durante la campaña. Por otro lado, Allen Iverson, tras salir de Philadelphia en 2007 después de toda una vida en ese equipo para jugar con Denver, quiso homenajear a su ex equipo cuando Denver visitó esa ciudad en marzo de 2008. Para ello, Reebok le grabó el acrónimo "THXPHILA”, que significa "Gracias Philadelphia”.

5. La siguiente categoría se refiere a una colaboración entre la marca y el jugador donde se homenajea a una persona cercana al jugador, 
normalmente por un fallecimiento repentino. Uno de los casos más destacados es el relativo al fallecimiento de Malik Sealy en mayo de 2000, cuando iba en coche a la fiesta de cumpleaños de su compañero Kevin Garnett, por obra de un conductor bebido que circulaba en sentido contrario. Garnett, muy afectado, quiso rendir tributo a su amigo grabando "2MALIK" (para Malik) en la lengüeta del modelo Adidas Garnett 3, con el que Garnett jugó la temporada siguiente y que se vendió con ese estampado al gran público. Algo similar realizó ha realizado DeAndre Jordan, con su modelo Under Armour Micro G Funk, donde homenajea a su amigo Tobi Oyedeji, jugador de la Texas A\&M University, que falleció en accidente de coche en 2010. En sus zapatillas está grabado "RIP TOBI" en la suela interior. En otras ocasiones, en vez de grabar una zapatillas los jugadores se hace grabaciones en la piel, como el caso de Courntey Lee, y su tatuaje en el brazo "RIP Danny Rumph", en homenaje por su temprana muerte cuando era jugador de Western Kentucky University en 2005. Lleva también su número en las zapatillas.

6. La sexta categoría se relaciona con la implicación del jugador en el propio diseño de las zapatillas que apadrina, es decir, ir más allá de ser un mero prescriptor de la marca para participar en la confección del producto. Esta estrategia es utilizada por las firmas para añadirle valor al producto, ya que al participar el jugador en su diseño, se transmite una imagen de mayor calidad y confianza, además de mantener al jugador con una vinculación más fuerte a la marca. En 1979, Rick Barry participó en la creación de las Adidas Top Ten, probablemente el primer caso conocido en baloncesto de esta forma de colaboración entre jugador y marca, algo que Adidas explotó convenientemente en su publicidad. Hasta esa fecha, algunas enseñas, como Nike en sus inicios, utilizaban el feedback de los atletas para realizar mejores en las zapatillas. El mítico entrenador y co-creador de Nike, Bill Bowerman, era el encargado de unir esas opiniones a su talento como innovador, con el fin de mejorar las zapatillas que la empresa de Oregon importaba de Japón. Actualmente, la implicación de ciertos jugadores en el diseño es mucho 
más común, desde Kobe Bryant y sus zapatillas de caña baja Nike Zoom IV, a Steve Nash y sus ecozapatillas Trash Talk en 2008. Estas últimas son un calzado en edición limitada hecho con material reciclable, y en el que Nash, un ferviente defensor del ecologismo y la sostenibilidad ha participado en el diseño, y también obviamente en la promoción. En España, por ejemplo, hace varias décadas Epi también se implicó en el diseño de su propio modelo para Adidas.

7. Zapatillas personalizadas desde el molde de diseño. Gracias a la tecnología de escaneado digital del pie, algunas empresas, como Digitoe, realizan moldes personalizados para cada pie, y además pueden realizar desarrollos artísticos sobre las superficies del calzado. En baloncesto, este tipo de "zapato a medida" se suele hacer con la colaboración de la marca que viste al jugador. En cualquier caso, lo importante es que existen herramientas para que el jugador tenga la zapatilla que realmente quiere. Y esto no es baladí, porque, por ejemplo, en los 80 el jugador Jose Luis Llorente llegó a "desfigurar" modelos de zapatillas con la asistencia de un zapatero, para convertir modelos de caña alta en baja, y así jugar más cómodo, y Fernando Romay a finales de los 70 le recortó la puntera a las Converse Chuck Taylor, que su compañero americano Walter Szcerbiak le había prestado, para así dejarle sitio a los dedos de su ingente pie. Esto ya no ocurre en la actualidad para los grandes jugadores, como Kobe Bryant por ejemplo, que pueden pedirle a la marca modificaciones de diseño, como que rasure un par de milímetros la parte de atrás de la zapatillas, en aras de conseguir un mayor rendimiento (Ballard, 2009), aunque las zapatillas "a medida" ya fueron una constante en España desde los 80, donde el propio Romay tuvo modelos de Adidas y Uliana Semenova de J'hayber.

Por tanto, las marcas pueden utilizar estas formas de proceder para implicar a los jugadores más aún con el producto, mejorar el vínculo relacional con la marca, y aprovechar la imagen de los jugadores para optimizar la comercialización de esos productos. Además, los jugadores también 
aprovechan su relación con las marcas, para proyectar ciertos valores, ideas o emociones personales.

\subsection{Formas de personalización no controladas por la marca}

Básicamente, hay dos formas de personalización no controladas por la marca:

1. Personalización de los modelos que algunas marcas permiten customizar a través de internet. El caso más conocido es el de Nike iD, el cual es utilizado no sólo por el gran público, sino por muchos jugadores profesionales para obtener zapatillas únicas. Así, por ejemplo, la plantilla del CB Valladolid, personalizó el modelo Huarache 2K4 durante la temporada 2010-2011. Algunos jugadores como Dumas pusieron los nombres de sus hijas, Martín el año en el que estaban compitiendo, y otros sus apodos.

2. Zapatillas personalizadas por otras empresas 0 personas que realizan un trabajo artístico sobre ellas. Existen webs especializadas, como www.clpstudio.com que realizan creaciones artísticas sobre cualquier tipo de calzado. En España, es conocido el caso del jugador Nacho Martín, quien encargó a un especialista (Joan SBS) este tipo de customización transformando un modelo totalmente liso en una zapa con diferentes colores, dibujos y letras. en las que se podía leer, "se puede comprar todo menos el talento" y "flow es lo que nunca tendréis", ambas frases son partes de canciones de rap español, con las que se identifica el jugador. En las zapatillas también estaba el logo de su grupo y su número. En otras ocasiones, ciertas herramientas de pintura pueden utilizarse para personalizar calzado, como por ejemplo Divermagic, un producto principalmente enfocado para niños pero que puede ser usado en una superficie como el calzado. En la NBA, por su parte, Baron Davis jugó varios partidos de play-off en 2012 con unas zapatillas Li-Ning BD Dooms, es decir, con su signature, pero pintadas por el artista de grafiti Big Doves, mostrando imágenes del skyline de Nueva York (su equipo). De forma similar, Dwyane Wade ha colaborado con el diseñador Mache para customizar sus Li-Ning Way of Wade en 2013. 
Sin embargo, de nuevo estas dos formas de personalización no son totalmente flexibles. Es cierto que un jugador puede, por ejemplo, diseñar su propio modelo con Nike iD para partidos determinados, pero ello no permite acciones espontáneas de personalización (normalmente se requieren de varios días para el envío). Además el número de caracteres para la impresión es limitado, así como el lugar de la zapatilla donde se puede insertar.

Por ello, los jugadores han buscado la forma de expresar mensajes de manera personal y espontánea a través, principalmente, de la rotulación de las zapatillas. Y este fenómeno es algo que las marcas ya conocen de sobra. De hecho, Adidas entre 1983 y 1985 lanzó al mercado varias versiones de las Adicolor, una zapatilla que incluía diversos rotuladores de diferente color para pintar el producto. Reebok, por su parte, en 2008, lanzó el modelo Talkin Krazy, que llevaba un rotulador negro, pero que contaba con la gran ventaja de que los mensajes se podían borrar y re-escribir fácilmente. Varios modelos más de Reebok incluyeron en rotulador también en sus cajas. No obstante, estos modelos tienen mayor calado entre el público general que entre los jugadores profesionales. Éstos últimos, quienes normalmente disponen de grandes cantidades de pares de zapatillas gratuitas, pueden pintar con rotulador permanente su zapatilla, independientemente del modelo que sea, y luego aparecer en otro partido con un nuevo par sin pintar. $Y$ es que, por ejemplo, jugadores como Ferrán Martínez pueden utilizar hasta 40 pares en una temporada.

Por tanto, ante el hecho de que los jugadores profesionales disponen de un gran número de pares de zapatillas cada temporada, pueden permitirse el lujo de escribir puntualmente mensajes en ellas, que sólo aparezcan en las ocasiones en las que lo requiere el jugador. Estas son las categorías fundamentales en las que se puede dividir los mensajes encontrados:

1. Religión: Los casos más representativos son los de Roger Powell y Stephen Curry. Ambos suelen jugar con sus zapatillas pintadas con versículos de la Biblia, en particular un pasaje de la Epístola a los 
Filipenses, capítulo 4, versículo 13: "Puedo hacer frente a todo pues es Cristo quien me da fuerzas" "“ can do all things through Christ which strenghteneth me"). Esos jugadores pueden escribir incluso un trozo de esa frase, como Curry, quien suele pintar "I can do all things...". Obviamente estos deportistas tienen unas fuertes convicciones cristianas, que como el caso de Curry, están muy arraigadas en su familia. La jugadora Betty Lennox, ha optado por grabarse ese mismo pasaje bíblico en la lengüeta de sus zapatillas, por lo que éste está permanentemente consigo.

2. Apoyo a compañeros: Sucede cuando un jugador muestra solidaridad con otros compañeros por diversos motivos. Por ejemplo, en el quinto partido de play-offs de 1997, ocurrió una pelea entre varios jugadores de Miami y New York. Tres jugadores de New York (Ewing, Houston y Ward) fueron suspendidos para el siguiente partido, y un compañero de equipo, John Starks, escribió sus nombres en una de sus zapatillas. En 2008, el jugador de Washington Antawn Jamison pintó el número 9 de su compañero Darius Songaila en el sexto partido de play-offs frente a Cleveland. Songaila fue suspendido para ese partido tras golpear a LeBron James en la cara en el partido anterior. Estas dos muestras de apoyo, tienen también una connotación de protesta ante la Liga, por sanciones que esos jugadores consideraban injustas. Sin embargo, otra forma de apoyo es la de recordar a compañeros lesionados, como hizo Dermarr Johnson en 2007, cuando se hizo referencia en su zapatilla a Carmelo Anthony, Kenyon Martin y JR Smith, lesionados de diferente gravedad.

3. Apoyo a políticos: La elección del primer presidente negro en EEUU en 2008, Barack Obama, fue celebrada por varios jugadores de la NBA, que pintaron sus zapatillas con su nombre. Así Chris Paul y Marresse Speights utilizaron el rotulador para expresar su apoyo a Obama de manera espontánea, mientras que como se ha comentado anteriormente, otros jugadores lo hicieron con zapatillas grabadas por las marcas que los vestían. 
4. Referencias a amigos y/o familiares: Ocurre cuando un jugador rinde tributo a amigos o familiares, en la mayoría de los casos porque han fallecido. El caso más paradigmático de esta categoría es el de Lamar Odom, quien cada vez que estrena unas zapatillas suele pintar los nombres de "Baby J", "Cathy" y "Grandma", en referencia al repentino fallecimiento de su hijo de 6 meses y medio, su madre que murió de cáncer cuando Odom tenía 12 años, y su abuela que lo cuidó después de esa desgracia. Esa referencia a personas queridas que han fallecido es habitual en ciertos jugadores americanos, que en algunos casos ponen la fecha y el nombre, o también "RIP" descanse en paz -. En otras ocasiones, un fallecimiento repentino hace que algunos jugadores rindan homenaje a esa persona en el partido siguiente a su muerte. Tal es el caso de Jose Calderón, y su referencia al periodista Andrés Montes, fallecido en 2010, escribiendo en sus Li-Ning “A. M. Descanse En Paz”, y también Chris Paul, que escribió el nombre de Brian en sus zapatillas, un chico de 8 años que murió de cáncer, y que era un gran seguidor de este jugador. Paul no pudo visitarlo en el hospital antes de que muriera. Mensajes similares escribieron los jugadores de Kentucky Wesleyan en sus zapatillas en el primer partido tras la muerte de su compañero Jeron Lewis, en enero de 2010.

5. Referencias a desgracias colectivas o sucesos impactantes: Sucede cuando un jugador hace su particular homenaje a sucesos trágicos que han conmocionado la sociedad. Por ejemplo, Kevin Durant en las semifinales del Mundial de 2010, señaló una fecha puntual en sus zapatillas por su significado especial en relación a una desgracia. Ese partido se jugó el 11 de septiembre, 9 años después de los atentados de las Torres Gemelas, y Durant escribió en sus zapatillas esa fecha (9-11-01), coincidiendo con el partido EUUU-Lituania. Otro ejemplo es la referencia que hicieron varios jugadores de la NBA tras la tragedia en la Escuela Elemental Sandy Hook, en Newtwon, Conneticut, en diciembre de 2012. Jugadores como Lebron James, Dwane Wade y Kevin Durant escribieron "Newton, CT" en sus 
zapatillas. Finalmente, ciertos jugadores también son sensibles a casos de violencia racial, como el caso de Trayvon Martin de 17 años, al que un vigilante de seguridad tiroteó quien por considerarlo sospechoso, únicamente por ser de raza negra y llevar una capucha. Ocurrió en Florida, en 2012. Además la policía no presentó cargos contra el vigilante. Tras ese suceso, los jugadores de Miami Heat, Chris Bosch, Lebron James y Dwyane Wade escribieron su nombre en las zapatillas y frases como "Queremos justicia" o "El podría ser nuestro hijo". La tragedia del Maratón de Boston en abril de 2013 también quedó reflejada en las zapatillas de Jeff Green, jugador de los Celtics, donde éste escribió \#BOSTRONSTRONG, el lema surgido para apoyar a la ciudad tras el atentado.

6. Referencias personales: Se refiere a cuando el jugador indica alguna característica personal, identificativa en la zapatilla. Por ejemplo, Óscar González a veces pintaba su número, 9, en sus zapatillas. La jugadora de la Universidad de Duke, Karima Christmas, en 2008, estuvo jugando con zapatillas completamente rotuladas, donde se podía leer, entre otras cosas, "LAX/HOU", en referencia a su lugar de nacimiento y residencia en ese momento.

7. Frases motivadoras: Aquí tienen cabida las frases motivadoras y significativas para los jugadores, que están fuera del universo religioso. Por ejemplo, la mencionada Karima Chirstmas Ilevaba escrito "The future belongs to those who believe in the beauty of their dreams" $y$ "our deepest fear is that we are powerful beyond measure", citas de Eleanor Roosevelt y Nelson Mandela, respectivamente. Por su parte, su compañera Wanisha Smith: "When I get the rock ur at my mercy" jerga que significa que significa algo así como "cuando cojo el balón no puedes hacer nada para pararme", o "I get buckets", conocida frase del famoso reportero Gust Johnson, conocido en EEUU por su estilo entusiasta, y que significa "Yo meto canastas".

8. Desafíos: En esta categoría están las alusiones a eventos anteriores que el jugador toma como un desafío para motivarse para un partido concreto. Un caso significativo ocurrió en los cuartos de final del 
Mundial 2010, cuando se enfrentaron EEUU y Rusia. El jugador americano Kevin Durant, pintó en sus Nike "1972", en alusión a la fecha en la que se celebraron los JJOO de Munich, donde se jugó aquella final entre EEUU y URSS que los soviéticos ganaron en la última jugada, y donde los americanos se sintieron víctimas de una injusticia deportiva (algo que objetivamente es muy discutible que así fuera).

Finalmente, dos últimas formas de personalización pueden identificarse, que están fuera de las categorías anteriores dadas sus especiales características:

1. Utilización de otros elementos integrados en las zapatillas: En este caso, no se estaría hablando de ediciones especiales de zapatillas con ciertos componentes tecnológicos incluidos, como la marca Shaq, del jugador Shaquille O'Neal, quien en 2005 diseñó una zapatilla con teléfono móvil incorporado, ni el modelo Adidas 1 de la marca alemana, con un microprocesador incorporado que ajusta la presión de la zapatilla al pie, sino de casos como el de Mark Jackson, quien ataba su anillo de casado en los cordones de su zapatilla izquierda en cada partido. Jackson lo hizo durante toda su carrera, desde que contrajera matrimonio en 1989. Otros jugadores como Tommy Mitchell o Kenny Smith también realizaban esa práctica. Este último jugador dejó de hacerlo después de que regalara una de sus zapatillas a un fan y olvidase que tenía su anillo atado a ella (Lukas, 2011).

2. Pintar la zapatilla para que parezca de otra marca. A finales de la década de los 80, la Federación Española de Baloncesto tenía un contrato firmado con Adidas, por el que los jugadores debían de llevar zapatillas de esa marca en los partidos de la selección. Hubo algunos jugadores, como Andrés Jiménez, que se sentían mucho más confortables con las zapatillas con las que jugaban habitualmente, y que eran de otra marca. Así, Jiménez solía pintar con un rotulador 3 bandas (el símbolo de Adidas) en sus zapatillas Air Force II de Nike. Precisamente Adidas ha sido históricamente 
muy perjudicada por este tema; un incidente similar ocurrió en la final de la FA Cup de fútbol en Inglaterra en 1966, donde varios jugadores del Liverpool fueron a una fábrica de zapatillas de la marca Gola, y se llevaron varios pares. Adidas les pagó para que todos llevaran su marca en esa final, pero varios de esos jugadores salieron al campo con las Gola pintadas a rotulador con 3 bandas...

El resumen esquemático de todas las formas de personalización descritas se puede visionar en la Figura $1^{9}$.

9 NOTA PARA LOS REVISORES: SI LOS REVISORES LO ESTIMAN OPORTUNO PODRÍA ADEMÁS AÑADIR UN SEGUNDO APÉDNICE IDENTIFICANDO A LOS NUMEROSOS JUGADORES DE BALONCESTO NOMBRADOS EN ESTE ARTÍCULO, EN ARAS DE FACILITAR AL LECTOR DATOS RELEVANTES SOBRE ELLOS. 
Figura 1. Esquema de las tipologías de personalización

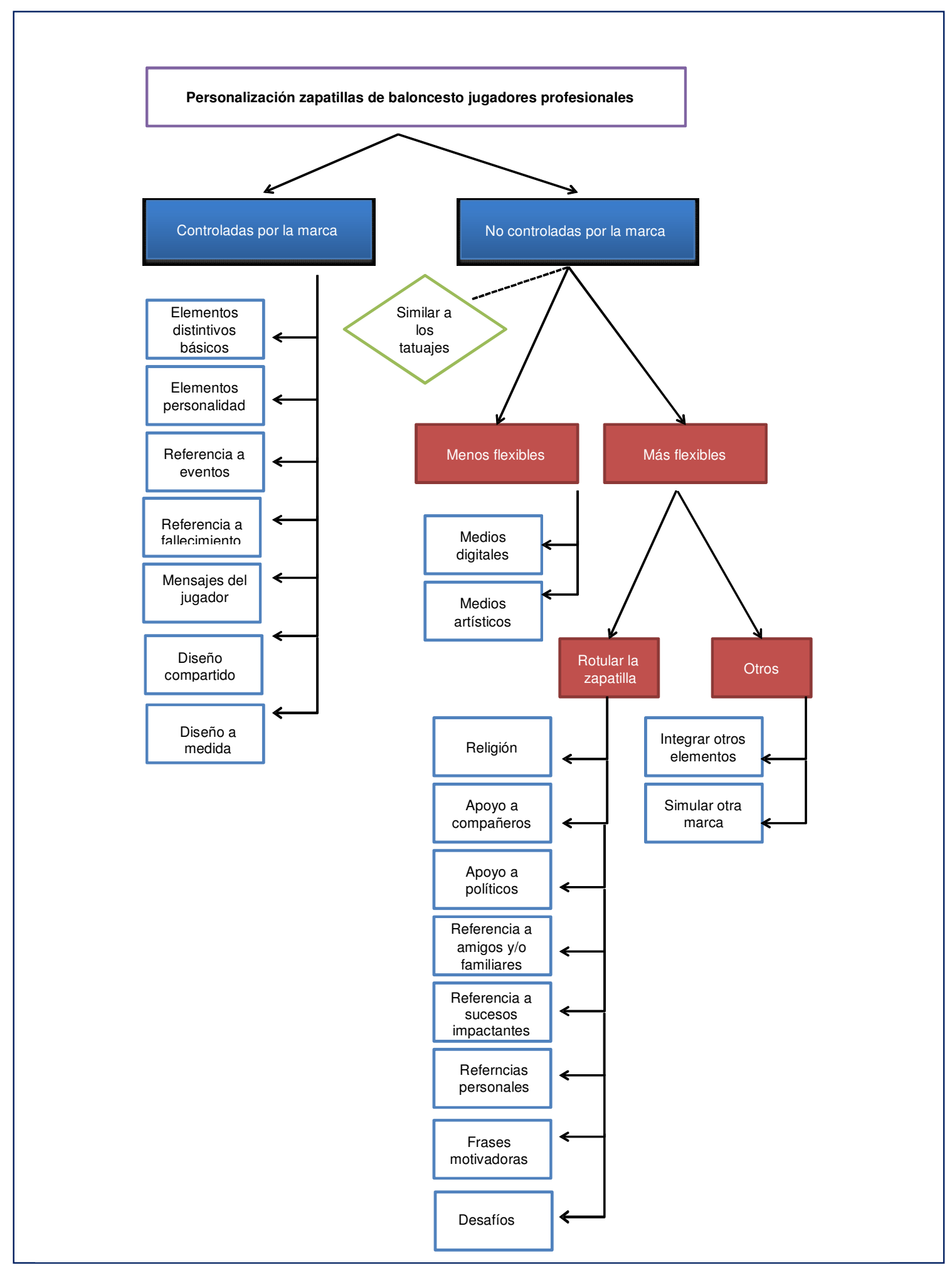




\section{DISCUSIÓN E IMPLICACIONES PARA LA GESTIÓN}

Esta investigación ha descrito explícitamente las diferentes formas en las que un jugador de baloncesto puede personalizar sus zapatillas, mostrando asimismo los diversos tipos de mensajes que en éstas se pueden transmitir, y relatando ejemplos concretos de cada uno de esas categorías.

Las zapatillas de baloncesto constituyen para algunos jugadores un medio de transmisión de mensajes similar a los tatuajes, pero con la ventaja de que en muchos casos se tiene más flexibilidad para proyectar el mensaje adecuado en el momento preciso. De hecho sólo en muy contados casos un jugador prefiere escribir un mensaje espontáneo en la piel frente a hacerlo en la zapatilla, como el caso del jugador Ryan Boatright de la Universidad de Connecticut, quien se pintó en la cara las iniciales "SH" del colegio tristemente masacrado en 2012. Lo habitual es, por tanto, utilizar las zapatillas como medio de expresión.

Actualmente, la tecnología es muy flexible y permite a los jugadores obtener zapatillas customizadas. Las marcas saben de la importancia de esta relación única con el jugador y tratan de potenciar el desarrollo de modelos que refuercen la identidad del jugador. Pero eso no basta, y algunos jugadores desean realizar acciones de comunicación fuera del paraguas de las marcas, y para ello pintan y rotulan sus zapatillas.

Así, las marcas pueden vislumbrar oportunidades de negocio en base a las tipologías que esta investigación muestra, aunque desde luego, no es nada fácil. Por ejemplo, jugadores de fuerte convicción religiosa utilizan versículos sagrados para motivarse. Una opción que tendrían las marcas es la de lanzar modelos específicos con alusiones religiosas. Investigaciones como las de Abou Bakar, Lee y Rungie, C. (2011) o Taylor, Halstead y Haynes (2010), sustentarían esta iniciativa. No obstante, ésta es una tarea muy arriesgada. El caso de Nike ejemplifica perfectamente ese riesgo: La marca de Oregón lanzó en 1998 el modelo Air Bakin, donde la tipografía estaba tan distorsionada que parecía leerse "Allah" en árabe. Rápidamente, ciertos colectivos musulmanes reaccionaron fervientemente en contra, y Nike tuvo que retirar el producto, 
aunque lo volvió a relanzar años más tarde con otro tipo de letra. Por tanto, si se acomete esta estrategia debe de hacerse con extrema sensibilidad, y tal vez una forma de hacerlo sería dando la oportunidad de que el consumidor pudiera obtener pegatinas con mensajes personalizados que fueran fácilmente despegables en la zapatilla, como ciertos adhesivos que se utilizan para marcar los zapatos de los niños en los colegios, por ejemplo. Otra opción sería que las marcas que permiten la customización, pusieran a disposición del público un catálogo de frases relacionadas con la religión, o con citas de célebres personajes. Esto probablemente incentivaría el uso de mensajes en las zapatillas, y se verían beneficiados tanto las marcas que personalizan de manera digital, como aquellas que ofrecen zapatillas con rotuladores incorporados, como Reebok. De hecho, ciertas webs cristianas, como www.jollynotes.com tratan de promover la lectura de la Biblia aprovechando las frases motivadoras que se pueden encontrar en ella para usar de inspiración a los deportistas. Por tanto, el poner a disposición del público un catálogo de citas y frases, podría beneficiar a las marcas.

Dado que hay jugadores profesionales, y también esto es extensible al gran público, que desean llevar consigo símbolos de su identidad individual y social, las marcas de zapatillas de baloncesto podrían inspirarse en los productos de la marca Archport. A comienzos de la década de 2000, esta marca lanzó al mercado zapatillas deportivas y sandalias con una suela móvil y con diversas hendiduras. Esa suela se podía sacar parcialmente de la zapatilla y en ella meter anillos, llaves o tarjetas de crédito. La idea estaba inspirada en la necesidad de algunas personas de llevar consigo esos objetos cuando no pueden disponer de bolsos u otro tipo de compartimentos independientes para realizar una actividad, como correr o ir a la playa, por ejemplo. Es cierto que el negocio de Archport se centra últimamente en las sandalias, y la línea de zapatillas deportivas parece que no ha funcionado especialmente bien, pero la idea sigue siendo atractiva para las grandes marcas de calzado de baloncesto. ¿Tal vez algún compartimento para insertar un anillo, un crucifijo, etc.? No es una idea nueva, ni mucho menos: En 1979, nació Kangaroos en Estados Unidos, una marca que se caracterizaba por la inclusión de un pequeño bolsillo 
en sus modelos. Esta marca, llegó a realizar zapatillas de baloncesto en los 80 y primeros 90, y tuvo su momento álgido cuando Clyde Drexler llevó uno de sus modelos en 1990. Poco después desaparecieron del universo del baloncesto, y ahora se enfocan más en la moda urbana y en el running. Tienen una patente (al igual que Archport), que protege su innovación, por lo que si otra marca quiere lanzar un modelos con bolsillos, tiene que pedir autorización a Kangaroos.

Por tanto, la clave podría estar en realizar un producto que permitiera la inclusión de algún objeto personal, ya sea con dispositivos en la suela o en cualquier otra parte de la zapatilla, pero que al mismo tiempo la zapatilla no perdiera un ápice de sus características técnicas. Esa posible disfunción, es lo que probablemente llevó a Archport a dejar de lado, al menos momentáneamente, la línea de calzado deportivo. Pero como añadir esos dispositivos está protegido por patentes, las marcas de zapatillas de baloncesto probablemente piensen que no es una estrategia adecuada. Recordemos que otras marcas han tratado de innovar en este aspecto, como Red or Dead y sus zapatos con reloj incorporados lanzados en 1987 (Design Museum, 2009), aunque la creatividad de las marcas choca en la mayoría de las ocasiones con las prestaciones técnicas que debiera tener unas zapatillas de baloncesto.

En 1993, L. A. Gear hizo unas zapatillas con luces intermitentes, innovación que ha inspirado otras tecnologías actuales Por ejemplo, ahora se pueden crear modelos que cambien de color con la luz del sol y ante fuentes de calor. Los modelos Nike 6.0 Dunk Color o Puma Basket CC son ejemplos de ello. Tal vez las marcas podrían usar la misma idea con la inclusión de mensajes, es decir, que la zapatilla llevara mensajes inscritos que salieran a la luz en función del calor.

Pero quizá una apuesta menos arriesgada es la de firmar acuerdos de colaboración con empresas o artistas dedicados a la personalización, con el fin de que el consumidor obtenga una ventaja diferencial al comprar una marca determinada y personalizarla. Otra opción es incentivar desde las propias webs 
de las marcas la personalización manual y "casera" de las zapatillas que venden, colgando vídeos acerca de los procedimientos para poder hacer tal personalización de manera fácil y en casa.

La co-creación de innovaciones por parte de marcas y consumidores puede ser un campo muy atractivo de explotar en el futuro de las zapatillas de baloncesto, como indican Fuller, Jawecki y Mühlbacher (2007). Los consumidores pueden ser muy innovadores y desarrollar nuevos productos a través de las comunidades on-line. En otros sectores, como el del motor por ejemplo, marcas como Harley-Davidson promueve entre usuarios de la marca la personalización de sus motocicletas para luego tomar algunas de esas ideas en el proceso de desarrollo de nuevos modelos. $Y$ es que incluso aunque esas ideas no fueran luego consideradas por la marca, el mero reconocimiento del valor de la personalización que hace la marca y la propia comunidad on-line es ya suficiente incentivo para que los consumidores customizen el producto. Nike y su comunidad Niketalk es una buena prueba de ello. Otras webs, como Kicksguide.com organizan competiciones mensuales sobre diseño de zapatillas, que son muy apreciadas por los aspirantes a diseñadores profesionales. Como se ha indicado anteriormente, las marcas podrían extender el hábito de la personalización al gran público ofreciendo guías y consejos sencillos para personalizar sus zapatillas en casa.

El cómo los jugadores profesionales personalizan sus zapatillas no es más que un reflejo de cómo lo hace el gran público. Si las marcas deben establecer una relación one-to-one con el jugador en base a ofrecerle un producto único y diferenciado con el que se identifique y con el que pueda proyectar sus valores y personalidad, también lo deben hacer con el resto de consumidores. La restricción que tienen esos consumidores es que no disponen de material casi ilimitado, como los jugadores profesionales, por lo que la elección del producto y su personalización es todavía más importante. Como indicaba (García, 2003), los jugadores de baloncesto callejero en Estados Unidos llevan personalizando sus zapatillas desde los años 70. Por eso, para las marcas, poner a disposición del público instrumentos para customizar es esencial. Desde luego que Reebok Talkin Krazy en 2008 fue un producto que se hizo 
eco de las demandas del consumidor. Pero existen otras fórmulas en el mercado. De hecho, un año antes, en 2007, se lanzaron las zapatillas Graffeeti, enfocadas para los niños y adolescentes. Esta marca ofrece un producto innovador donde se puede dibujar y borrar con rotuladores de varios colores. Es decir, una especie de mezcla entre las Adicolor de Adidas de comienzos de los 80 (en éstas no se podía borrar el dibujo), y las Talkin Crazy de Reebok (éstas sólo llevan un rotulador negro). Tal vez, el lanzamiento al mercado de un producto así enfocado al baloncesto sería bastante atractivo, pero, una vez más, la patente (en este caso se le concedió en 2010 en Estados Unidos), es una barrera para el resto de marcas, que podrían plantearse la opción de firmar un acuerdo de colaboración con Graffeeti. De hecho Reebok tuvo que retirar sus zapatillas Talkin Krazy del mercado debido a la protección que Graffeeti obtuvo por su patente (J. Mulligan, comunicación personal, 13 febrero, 2012).

El jugador Darryl Dawkins, en 1982 jugó con una zapatilla de la marca Nike y otra de la marca Pony (Katz, 1994), debido a que quiso mantener el contrato con Nike cuando Pony le ofreció dinero por cambiar de marca. Asimismo, Ron Artest jugó con varias zapatillas de marcas distintas en cada pie en el All-Star de 2004 (Nike, Dada, Adidas...), aunque éste jugador no tenía en ese momento contrato con ninguna marca deportiva (Seize9, 2013), siguiendo una costumbre que ya realizaba en la Universidad de St. John. Al margen de esos casos particulares, las marcas ya se han dado cuenta de lo atractivo que puede ser para el consumidor llevar diseños diferentes en cada pie, caso por ejemplo de las Nike Zoom Kobe VII "Snake Pool", con las que Kobe Bryant ha jugado partidos en 2012 o de las Adidas T-Mac 3 que llevó Tracy McGrady en 2004. Los más recientes (2013), sin embargo, son el de los jugadores de la Universidad de Maryland, vestida por Under Armour, y cuyos jugadores calzan colores diferentes en cada pie, y las zapatillas de LeBron James conmemorativos de su cuarto premio MVP. Esta es otra vía por la cual las marcas podrían explotar la personalización, a través de la apuesta por diseños divergentes y la transmisión de mensajes diferentes para el pie izquierdo y para el derecho, y es también una opción que se ha probado en otros deportes, 
como el Maratón y las zapatillas que Nike personalizó para el corredor Mo Farah.

Otra opción es crear zapatillas personalizadas a jugadores profesionales cuyo diseño pueda ser perfectamente replicado por los consumidores a través de plataformas como NikeiD, por ejemplo. Es lo que hizo Nike en 2012 con las zapatillas de Jeremy Lin, el jugador de origen taiwanés que encandiló a la NBA en sus primeras apariciones. Nike diseñó una zapatilla para Lin que cualquier consumidor podía copiar a través de NikeiD (Seize9, 2012d). Esta es una forma de reforzar los vínculos de identidad entre el consumidor final y el jugador.

La personalización de zapatillas genera publicity, por lo que esa es una de las razones por las que las marcas se deben volcar en este tipo de productos customizados. La información sobre cualquier nuevo modelo personalizado es automáticamente amplificada por la ingente comunidad de webs y blogs especializados en zapatillas, además de, por supuesto, otros medios de comunicación convencionales (aunque estos últimos únicamente suelen recoger las noticias más sobresalientes a este respecto). Por ello, las marcas no sólo se involucran en la personalización de modelos para sus estrellas del baloncesto, sino también para otros personajes relacionados en mayor o menor medida con este deporte. Por ejemplo, como indica Geary (2012), Reebok le diseño una zapatilla a medida a lgor Voykivinsky, el hombre más alto de Estados Unidos $(2,38 \mathrm{~m})$, reconocido fan de uno de los equipos de la NBA: Minnesota Timberwolves. Este hombre llevaba años buscando mecenas que le costearan unas zapatillas, ya que el molde y escáner de su pie ascendían a 16000 dólares. Con un poco más de inversión (25000 dólares) Reebok le diseñó varias zapatillas personalizadas, con su nombre en la suela y con los colores de su equipo favorito, que la marca ha amortizado con creces por la generación de publicity. Otro caso es el de la personalización de un modelo de Nike Air Foamposite para el famoso director de cine Spike Lee, ferviente seguidor de los New York Knicks (Brillian, 2012), incluyendo el símbolo de su productora "40 Acres \& A Mule" y los colores de su equipo en la zapatilla. Lee ha acudido a ver partidos de su equipo calzando su modelo personalizado. 
Precisamente Spike Lee en colaboración con la marca Jordan (subsidiaria de Nike) diseñó unas zapatillas personalizadas para el presidente de Estados Unidos, Barak Obama (Scav, 2012), cuya afición por el baloncesto (especialmente por los Chicago Bulls) es notoria, estrategia que también ha seguido New Balance. Esta última marca es de las pocas enseñas que fabrica calzado deportivo en Estados Unidos, y su modelo personalizado incluía las palabras "Presidente", "Obama" y "Hechas en los Estados Unidos. Otras veces, una marca apuesta por un personaje célebre vinculado al baloncesto como el productor musical y jugador aficionado P. Miller, a quien Converse dedicó una línea de zapatillas en 1999, pero que pese a entrenar con varios equipos en la NBA, nunca llegó a debutar. En España, por su parte, las marcas están empezando también a emplear esta forma de proceder, como el caso de la marca valenciana J'hayber, que aunque en otro deporte -fútbol-, entregó unas zapatillas personalizadas (modelo Olimpo) con los colores de la bandera española al conocido aficionado Manolo "el del Bombo" para animar a la selección en la Eurocopa de 2012 (Martí, 2012).

Pero el recurso de la personalización como herramienta de marketing va incluso más allá de vestir celebridades del deporte o de cualquier ámbito. Tal es el caso de colaboración de Nike con el Hospital Infantil Doernbercher, en Oregón (Estados Unidos). Como indica Seize9 (2012b), en una charla casual descubrieron que muchos de los niños ingresados estaban también interesados en las zapatillas y Nike les propuso crear sus propios modelos personalizados. Los beneficios de la venta de esta serie ascienden a 4 millones de dólares y son donados al hospital. Un repaso a algunos de los diseños de esos niños muestra que su necesidad de expresar su identidad, en base a su historia y emociones, es similar a la de los baloncestistas profesionales. Por ejemplo, Chad Berg al caer de su kayak estuvo 90 minutos en aguas heladas. Este niño eligió un modelo Air Force One con los colores de su equipo, los Oregon Ducks y una frase en la plantilla "The people who turn out the best are those people who make the best out of the way things turn out". Finnigan Mooney, tras 13 operaciones de corazón, escribió "Thump Thump" por el sonido que hace su corazón, que está pintado sobre la zapatilla. El swoosh (símbolo de Nike), está 
cosido a la zapatilla recordando sus cicatrices. Grant Olsen, por su parte, ha reunido en un logotipo de la lengüeta de unas Free Run+ 2 muchas de sus pasiones, la guitarra eléctrica, sus iniciales y la frase "Do not fear or be dismayed", recuerdo de su leucemia. La niña Kylie Bell está recuperándose de un tumor cerebral y decoró unas Free Run+ 2 con telas parecidas a su camiseta favorita, un pájaro en la lengüeta y las palabras "Fuerza" y "Libertad" en los cordones. Finalmente, Oswaldo Jiménez, con un problema pulmonar, eligió su apodo, "Pollito", como lema de la zapatilla, colocando plumas grabadas en láser y los colores de México en la suela como recuerdo de su lugar de nacimiento. Por tanto, no sólo la elaboración de un producto personalizado es atractiva para su creador por la oportunidad de expresar su identidad a través de un lenguaje con diferente grado de simbología, sino que hay personas que se identifican con ese producto (precisamente por lo que trata de expresar) y lo adquieren para reforzar su propia identidad.

Por esa misma razón ya hay webs que venden camisetas con el dibujo de tatuajes de jugadores de baloncesto, como www.redbubble.com, donde se puede encontrar, por ejemplo, una camiseta con el tatuaje del cabello medieval que el jugador Nikola Pekovic lleva en el brazo izquierdo (Sardinero, 2012). Pekovic, al igual que otros muchos jugadores tatuados, reconoce que sus tatuajes representan lo que él es, además de todo lo que le ha costado llegar a donde ha llegado. Y el lenguaje empleado para tal expresión es también atractivo para aquellos consumidores que se identifican con el mensaje. De ahí la importancia y el interés que marcas, jugadores y consumidores finales muestran por la personalización de las zapatillas.

Finalmente, el empaquetado de las zapatillas es otra fuente atractiva para la personalización. Por ejemplo, Nike personalizó unas Air Force One para Dirk Nowitzki empaquetando las zapatillas en una caja especial, donde se grabó una frase inspiradora del jugador alemán "Todos los sueños son una locura hasta que se hacen realidad. Dirk lo hizo". La grabación de mensajes personales en las cajas de zapatillas podría potenciar la atracción por el producto. 
En conclusión, esta investigación ha mostrado las numerosas formas en las que un jugador puede expresarse a través de sus zapatillas, desde colaboraciones con la marca que lo apadrina a expresiones espontáneas personales fuera del amparo de la enseña que lo viste. Esto da una idea de la importancia que tienen las zapatillas como señas de identidad del jugador, no sólo profesional, sino también aficionado, y cómo las marcas aprovechan esta coyuntura para lanzar productos personalizados. Un estudio en profundidad de las tipologías de personalización de zapatillas de baloncesto, como el realizado en este trabajo, sirve además para que las marcas tengan un registro de acciones de personalización, y encuentren oportunidades de explotar este fenómeno con la creación de nuevos modelos. 


\section{Agradecimientos:}

Esta investigación está en deuda con la Fundación Pedro Ferrándiz (en especial con John Larkin), Mario Hernando (Director de Comunicación de la Liga ACB), Franco Pinotti (Departamento de Scouting de la Liga ACB y ex Director de Nuevo Basket), Jordi Román (Coordinador de Nuevos Proyectos de la FEB), Paco Torres (Director de Gigantes del Basket).

Agradezco enormemente también la deferencia de varios jugadores y ex jugadores de baloncesto por acceder a ser entrevistados: Nino Buscató, Roger Esteller, Aíto García Reneses, Andrés Jiménez, Jose Luis Llorente, Juanma L. Iturriaga, Óscar González, Nacho Martín, Ferrán Martínez, Juan A. San Epifanio y Luis Miguel Santillana.

Asimismo, gracias a www.seizenine.blogspot.com.es, www.suelasdegoma.com, y www.lovezapas.es.

Finalmente, gracias a Steven Caudill, Julián Felipo, Alan Hahn, Diane Halstead, Jose Luis López (Kangaroos), Luis Mendiola, Paco Rengel, Juan Francisco Escudero, Jim Mulligan (Graffeeti), Matt Potts (Archport), Jordi Robirosa, Eduardo Schell, Valerie Taylor, CB Murcia y CB Valladolid por facilitar el acceso a diferentes informaciones relacionadas con esta investigación. 


\section{REFERENCIAS}

Belk, R. W. (1988). Possesions and the extended self. Journal of Consumer Research, 15, 139-168.

Brace-Govan, J., \& de Burgh-Woodman, H. (2008). Sneakers and street culture: A postcolonial analysis of marginalized cultural consumption. Consumption Markets \& Culture, 11 (2), 93-112.

Brillian, J. (2012). Spike Lee's Nike Air Foamposite One "Knicks" Shoes. Counterkicks.com. Disponible en: http://counterkicks.com/2012/05/spike-leenike-air-foamposite-one-knicks-shoes/ [Acceso 10 Enero 2013]

Design Musseum. (2009). Fifty shoes that changed the world. New York: Conran Octopus

Dimofte, C. V. \& Yalch, R. F. (2011). The mere association effect and brand evaluations. Journal of Consumer Psychology, 21, 24-27.

Falk, D., 2009. The bald truth. Pocket books.

Firmin, M. W., Tse, L. M., Foster, H. \& Angelini, T. (2008). Christian student perceptions of body tattoos: A qualitative analysis. Journal of Psychology and Christianity, 27 (3), 195-204.

Frisch, A. (2009). The story of Nike. Saunders Book Company: Collingwood.

Fuller, J., Jawecki, G. \& Mühlbacher, H. (2007). Innovation creation by online basketball communities. Journal of Business Research, 60 (1), 60-71.

García, B. (2003). Where'd you get those? Testify Books: New York.

García, G. (2012). El "35”, el mejor homenaje al hombre que cambió la vida de Durant.

Disponible

en:

http://www.marca.com/2012/06/01/baloncesto/nba/noticias/1338535732.html [Acceso 31 Enero 2013]

Geary, M. (2012). Tallest U.S. man gets $\$ 25 \mathrm{~K}$ sneakers from Reebok USA Today. Disponible en:

http://www.usatoday.com/story/gameon/2012/10/20/igor-vovkovinskiytallest-us-man-receives-25k-shoes/1646439/ [Acceso 20 Enero 2013]

Katz, D. (1994). Just Do It: The Nike Spirit in the Corporate World. Adams Media Corporation, Hollbrook, MA. 
Kelly, S. W., \& Tian, K. (2004). Fanatical consumption. An investigation of the behaviour of sports fans through textual data. En Kahle y Riley (Ed), Sports marketing and the psychology of marketing communication, $27-65$.

Lukas, P. (2011). On the field, wearing a band of gold. Disponible en: http://espn.go.com/espn/page2/story//id/7182495/uni-watch-talks-ben\begin{tabular}{ll} 
roethlisberger-other-athletes-wearing-wedding-rings-field & [Acceso \\
\hline
\end{tabular} Febrero 2012]

Martí, L. (2012). Manolo "el del Bombo" ya tiene sus zapatillas para la Eurocopa. Las Provincias. Disponible en: http://www.lasprovincias.es/20120528/deportes/futbol/manolo-bombocalzado-eurocopa-201205281720.html [Acceso 12 Enero 2013]

Morales, J. F., Moya, M.C., Gaviria, E. \& Cuadrado, I. (eds.) (2007). Psicología Social, Tercera Edición, Madrid: McGrawHill

Neuendorf, K. A. (2002). The content analysis guidebook. Sage Publications. Thousand Oaks, London.

O'Cathain, A., Murphy, E., \& Nicholl, J. (2008). The quality of mixed methods studies in health services research. Journal of Health Services Research Policy, 13 (2), 92-98.

Packaged Facts. (2009). The global footwear market.

Riffe, D., Lacy, S. \& Fico, F. (2008). Analyzing media messages. Using quantitative content analysis in research. Taylor \& Francis Group. New York.

Rovell, D. (2008). The Revolutionary Kobe Zoom Cut Shoe. Disponible en: www.cnbc.com/id/28180818/The Revolutionary Kobe Zoom Cut Shoe [Acceso 12 Febrero 2012]

Sardinero, D. (2012). El último socio de Ricky llegó del infierno y allí es venerado: Nikola Pekovic. Disponible en: http://www.marca.com/blogs/basket-and-roll/2012/02/09/el-ultimo-socio-dericky-llego-del.html [Acceso 31 Enero 2013]

Scav, (2012). President Obama Gets His Own Customized Air Jordan Spizikes. Disponible en: http://live.driays.com/index.php/2012/01/21/president-obamagets-his-own-customized-air-jordan-spizikes/ [Acceso 10 Enero 2013] 
Seize9, (2012a). Kickstories: Jordan XX. Disponible en: http://seizenine.blogspot.com.es/2012/03/kickstories-jordan-xx.html [Acceso 31 Enero 2013]

Seize9, (2012b). Nike Doernbecher 9. Disponible en: http://seizenine.blogspot.com.es/2012/10/nike-doernbecher-9.html [Acceso 31 Enero 2013]

Seize9, (2012c). Nike Hyperfuse Low iD. Disponible en: http://seizenine.blogspot.com.es/2012/02/nike-hyperfuse-low-id.html [Acceso 31 Enero 2013]

Seize9, (2012d). Nike Lunar Force One. Disponible en: http://seizenine.blogspot.com.es/2012/11/nike-lunar-force-one.html [Acceso 31 Enero 2013]

Seize9, (2012e). Picstories: Puma Clyde, zapatillas con nombre de gangster.

Disponible en: http://seizenine.blogspot.com.es/2012/04/picstories-pumaclyde-zapatillas-con.html [Acceso 31 Enero 2013]

Seize9, (2013). Cosas que nunca te dije: los cambios de Ron Artest en el All Star. Disponible en: http://seizenine.blogspot.com.es/2013/02/cosas-quenunca-te-dije-los-cambios-de.html [Acceso 31 Enero 2013]

Sfia.org, (2008). Basketball: Alive \& Well Around The World- SGMA. Dispobible en: http://www.sfia.org/press/76 Basketball\%3A--Alive-\%26-Well-AroundThe-World--SGMA-Figures-Showcase-Strength-of-\%91Hoops\%92-in-the-

U.S. [Acceso 10 Noviembre 2011]

Slater, J., \& Lloyd, C. (2004). It's gotta be the shoes: Exploring the effects of relationships of Nike and Reebok sponsorship on two college athletic programs. En Kahle y Riley (Ed), Sports marketing and the psychology of marketing communication, 191-210.

Stallen, M., Smidts, A., Smit, G. Klucharev, V., Fernández, G. \& Rijpkema, M. (2009). Celebrities and Shoes on the Female Brain: The Neural Correlates of Product Evaluation in the Context of Fame (26 2009, 08). ERIM Report Series Reference No. ERS-2009-048-MKT. Disponible en SSRN: http://ssrn.com/abstract $=146260$

Strasser, J. B. \& Becklund, L. (1991). The story of Nike and the men who played there. Harcourt Brace Jovanovich: San Diego. 
Styles, U. (2005). Sneakers. The complete collectors' guide. Thames \& Hudson. Summers, M. B. (2013). Behind the numbers. NBA.com. Diponible en: http://www.nba.com/features/behind the numbers.html. [Acceso 28 Febrero 2013]

Vanderbilt, T. (1998). The sneaker book. Anatomy of an industry and an icon. The New Press. New York

Vesanen, J. (2007). What is personalization? A conceptual framework. European Journal of Marketing, 41 (5/6), 409 - 418

Yoh, T. (2005). Parent, peer and TV influences on American teens' athletic shoes purchasing. International Journal Sport Management and Marketing, $1,1 / 2,180-189$. 
APÉNDICE 1. Características de la muestra de publicaciones periódicas

\begin{tabular}{|c|c|c|c|c|c|}
\hline Revista & $\begin{array}{c}\text { Números } \\
\text { analizado } \\
\text { s } \\
\end{array}$ & $\begin{array}{l}\text { Números } \\
\text { publicados }\end{array}$ & Fecha inicio & $\begin{array}{l}\text { Fecha } \\
\text { finalización }\end{array}$ & $\begin{array}{l}\text { Números } \\
\text { que faltan }\end{array}$ \\
\hline 5 Todo Baloncesto ${ }^{1}$ & 22 & 22 & Enero 1980 & Diciembre 1982 & Ninguno \\
\hline $\begin{array}{l}70 \text { años de basket en } \\
\text { España }^{2}\end{array}$ & 20 & 20 & $\begin{array}{c}\text { Noviembre } \\
1989 \\
\end{array}$ & Marzo 1990 & Ninguno \\
\hline ACB Publicaciones & 27 & 27 & 1986 & 2011 & Ninguno \\
\hline American Basket & 12 & 15 & Junio 1997 & Septiembre 1998 & $\begin{array}{c}11,12 y \\
14\end{array}$ \\
\hline American Superbasket & 26 & 26 & Diciembre 2002 & Diciembre 2003 & Ninguno \\
\hline Basket 16 & 58 & 58 & Julio 1988 & Agosto 1989 & Ninguno \\
\hline Basket 16 Profesional & 1 & 1 & Mayo 1989 & Mayo 1989 & Ninguno \\
\hline Basket Americano & 5 & 5 & 2010 & 2011 & Ninguno \\
\hline Basket FEB & 76 & 84 & Marzo 1999 & 2009 & $\begin{array}{l}8,22,24,34 \\
, 52,53,66 \\
67\end{array}$ \\
\hline Basket Life & 21 & 21 & Marzo 2008 & Noviembre 2009 & Ninguno \\
\hline BasketMe (Guías) & 14 & 14 & 2009 & 2011 & Ninguno \\
\hline Crossover Magazine & 4 & 4 & 2009 & 2011 & Ninguno \\
\hline Cuadernos del Entrenador & 17 & 17 & 1990 & 1993 & Ninguno \\
\hline Don Basket & 57 & 57 & Abril 1989 & Diciembre 1993 & Ninguno \\
\hline $\begin{array}{l}\text { Don Basket (números } \\
\text { extras) }\end{array}$ & 6 & 7 & 1984 & 1992 & 1 \\
\hline El Periódico del Basket & 29 & 29 & $\begin{array}{c}\text { Noviembre } \\
1999 \\
\end{array}$ & Junio 2000 & Ninguno \\
\hline Estrellas del Baloncesto & 16 & 16 & Octubre 1987 & Enero 1988 & Ninguno \\
\hline Estrellas del Basket & 6 & 6 & Octubre 1987 & Noviembre 1987 & Ninguno \\
\hline Estrellas del Basket 16 & 32 & 32 & $\begin{array}{c}\text { Noviembre } \\
1987\end{array}$ & Junio 1988 & Ninguno \\
\hline FIBA Basket (1aㅡ ed,) & 11 & 11 & Diciembre 1991 & Octubre 1992 & Ninguno \\
\hline FIBA Basket (2o ed.) & 29 & 29 & $\begin{array}{c}\text { Noviembre } \\
1993 \\
\end{array}$ & Mayo 1996 & Ninguno \\
\hline Gigantes del Basket $^{4}$ & 1364 & 1364 & $\begin{array}{c}\text { Noviembre } \\
1985 \\
\end{array}$ & Diciembre 2011 & Ninguno \\
\hline Inside the NBA & 3 & 11 (7 a 17) & $\begin{array}{l}\text { Noviembre } \\
1994\end{array}$ & Diciembre 1996 & $\begin{array}{c}7,8.9,10,1 \\
1, \\
12,13,15 \\
\end{array}$ \\
\hline Jugón Basket & 1 & 1 & Febrero 2010 & Febrero 2010 & Ninguno \\
\hline $\begin{array}{l}\text { Marca (Guías) (no se } \\
\text { incluyen las guías oficiales } \\
\text { ACB) }\end{array}$ & 0 & 4 & 2006 & 2010 & $\begin{array}{c}\text { Guías de } \\
\text { diferentes } \\
\text { eventos }\end{array}$ \\
\hline $\begin{array}{l}\text { Marca 3,05 (tengo } \\
\text { controlado hasta el } 129 \text { pero } \\
\text { posiblemente falta alguno } \\
\text { más) }\end{array}$ & 112 & 129 & 2003 & 2006 & $\begin{array}{c}\text { Del } 113 \text { al } \\
129\end{array}$ \\
\hline
\end{tabular}




\begin{tabular}{|c|c|c|c|c|c|}
\hline Mi Baloncesto $^{2}$ & 52 & 52 & 1985 & 1986 & Ninguno \\
\hline Monográficos NBA ${ }^{5}$ & 12 & 23 & Octubre 1994 & Julio 2001 & $\begin{array}{r}1,8,9,12,1 \\
3,14,15,17 \\
18,20,22 \\
\end{array}$ \\
\hline Nuevo Basket & 206 & 206 & Octubre 1980 & Mayo 1991 & 124 \\
\hline $\begin{array}{l}\text { Nuevo Basket semanal ( } 1^{\circ} \\
\text { ed.) }\end{array}$ & 29 & 29 & $\begin{array}{c}\text { Noviembre } \\
1986\end{array}$ & Junio 1987 & Ninguno \\
\hline $\begin{array}{l}\text { Nuevo Basket semanal ( } 2^{0} \\
\text { ed.) }\end{array}$ & 25 & 25 & Octubre 1990 & Abril 1991 & Ninguno \\
\hline Revista Basketblog & 4 & 4 & Enero 2011 & Diciembre 2011 & Ninguno \\
\hline Revista Oficial NBA & 214 & 228 & $\begin{array}{l}\text { Noviembre } \\
2001\end{array}$ & Diciembre 2011 & $\begin{array}{c}154,164,1 \\
66,204,22 \\
3\end{array}$ \\
\hline Superbasket ( $1^{\circ}$ ed.) & 27 & 27 & Marzo 1986 & Mayo 1988 & Ninguno \\
\hline Superbasket ( $2^{\circ}$ ed.) & 14 & 14 & Junio 1988 & Agosto 1989 & Ninguno \\
\hline Superbasket ( $3^{\circ}$ ed.) & 209 & 209 & Octubre1989 & Febrero 1994 & Ninguno \\
\hline Suplementos digitales FEB & 269 & 269 & 2005 & 2010 & Ninguno \\
\hline Tiro Adicional & 41 & 41 & Febrero 2011 & Diciembre 2011 & Ninguno \\
\hline Viva Basket & 2 & 2 & Diciembre 1992 & Enero 1993 & Ninguno \\
\hline XXL Basketball ${ }^{6}$ & 47 & 57 (18 a 74) & Enero 1997 & Octubre 2001 & $\begin{array}{c}22,25,27,3 \\
5,40,42,43 \\
, 46,48,70 \\
\end{array}$ \\
\hline Zona 2-3 & 2 & 2 & 2011 & 2011 & Ninguno \\
\hline Total & 3122 & 3198 & 1 enero 1980 & $\begin{array}{c}31 \text { diciembre } \\
2011\end{array}$ & 76 \\
\hline
\end{tabular}

'Se editaron 34 números, pero 22 sólo en el periodo de análisis (a partir de enero de 1980), ya que la revista comenzó en junio de 1978.

${ }^{2}$ Se vendía en fascículos semanales

${ }^{3}$ Los números extras de Don Basket pueden tener errores en su cuantificación. Ha sido imposible identificar los números publicados, considerando además, que hubo un reinicio en la numeración en los años 90 . Probablemente falta uno, o unos pocos números más.

${ }^{4}$ Comprende los diferentes cambios de nombre que ha sufrido la revista en su andadura (incluyendo Gigantes del Superbasket y Gigantes del Basket Teledeporte). Esta revista comenzó su periplo en 1985 a través de la editorial Hobby Press, y no tiene nada en común con una revista también llamada "Gigantes" editada en Barcelona en 1979 y que tuvo una vida muy corta (7 números, todos en ese año). Hobby Press realizó una consulta con el Registro de Marcas para comprobar si esa cabecera seguía existiendo, pero desde hacía tres años estaba liberada, por lo que la revista se lanzó al mercado con el nombre de "Gigantes del Basket" (P. Torres, comunicación personal, Mayo 26, 2011).

${ }^{5}$ Una parte de los Monográficos NBA (del mismo grupo de la Revista Oficial NBA) fueron editados por el equipo de Inside the NBA, y corresponden a los primeros números de esa revista. Desde que Inside the NBA fue editado por el equipo de Rob Dugdale y posteriormente Lee Berry, los Monográficos NBA fueron independientes.

${ }^{6}$ El primer número de la revista XXL Basketball es el número 18, ya que es una continuación del trabajo editorial de la revista Inside the NBA, de la que se publicaron 17 números. Por tanto, XXL Basketball publicó 57 números (del 18 al 74). 